\title{
Low frequency observations of cosmic ray air shower radio emission by CODALEMA/EXTASIS
}

\author{
Antony ESCUDIE ${ }^{* 1}$, D; Charrier ${ }^{1,3}$, R.Dallier ${ }^{1,3}$, D.García-Fernández ${ }^{1}$, A.Lecacheux ${ }^{2}$, \\ L.Martin $^{1,3}$ and B.Revenu ${ }^{1,3}$ \\ ${ }^{1}$ Subatech, Institut Mines-Telecom Atlantique, CNRS, Universite de Nantes, Nantes, France \\ ${ }^{2}$ CNRS-Observatoire de Paris, Meudon, France \\ ${ }^{3}$ Unite Scientifique de Nancay, Observatoire de Paris, CNRS, PSL, UO/OSUC, Nancay, France \\ E-mail: antony.escudiedsubatech.in2p3.fr
}

\begin{abstract}
Over the years, significant efforts have been devoted to the understanding of the radio emission of extensive air shower (EAS) in the range [20-200] MHz. Despite some studies led until the early nineties, the [1-10] MHz band has remained unused for 20 years. However, it has been measured by these pioneering experiments and suggested by theoretical calculations that EAS emit a strong electric field in this band and that there is evidence of a large increase in the amplitude of the radio pulse at lower frequencies. The EXTASIS project, located within the radio astronomy observatory of Nançay and supported by the CODALEMA instrument, aims to study the [1-10] MHz band, and especially the so-called "Sudden Death" contribution, the expected radiation electric field created by the particles that are stopped upon arrival to the ground. We present our instrumental setup, the objectives of the EXTASIS project and our first results.
\end{abstract}

35th International Cosmic Ray Conference - ICRC2017

10-20 July, 2017

Bexco, Busan, Korea

${ }^{*}$ Speaker. 


\section{Introduction}

It is a well known fact that the coherent radio emission during the development of air shower has two main origins: geomagnetic and charge excess mechanisms $[1,2,3]$. The resulting emission appears as a fast electric field transient lasting few tens of ns, and this can be detected by large bandwidth $(\sim 100 \mathrm{MHz})$ antennas and acquisition systems. Man-made broadcasting at low and medium frequencies constrains the common observation in the range $[30-80] \mathrm{MHz}$, but several detections at low frequencies have been realized in the 70's and up to the 90's, and a conclusion can be drawn from these observations: extensive air showers (EAS) undoubtedly emit a strong electric field at low frequencies. At that time, several experiments $[4,5,6,7]$ have shown that when frequency decreases, there is a clear evidence of a strong increase of the radio pulse amplitude. We have therefore chosen to reinvestigate low frequency measurements by implementing in the CODALEMA experiment low frequency antennas and using the SELFAS simulation code [8] to highlight a possible new emission mechanism. Figure 1 shows the simulation with SELFAS code of the electric field strength as a function of time, recorded by an antenna in vertical polarization located $300 \mathrm{~m}$ to the shower core (in $\mu \mathrm{V} \cdot \mathrm{m}^{-1}$ ) for a vertical shower initiated by a proton at $0.1 \mathrm{EeV}$.

On the full-band trace (blue line), the negative peak at $0 \mathrm{~ns}$ is due to is due to the shower development in the air. The second, positive peak around $1 \mu$ s has been interpreted as the effect of the coherent deceleration of the shower front hitting the ground: the sudden death pulse (SDP) $[9,10]$. The sudden death pulse arrives $1 \mu$ after the normal pulse, which is consistent with the propagation time to antenna $(300 \mathrm{~m})$. By filtering in different frequency bands, we show that both signals are still detectable for frequencies below $<10 \mathrm{MHz}$, suggesting the use of low frequency antennas: this is the goal of the EXTASIS (EXTinction of Air-Shower Induced Signal) project. In the following,

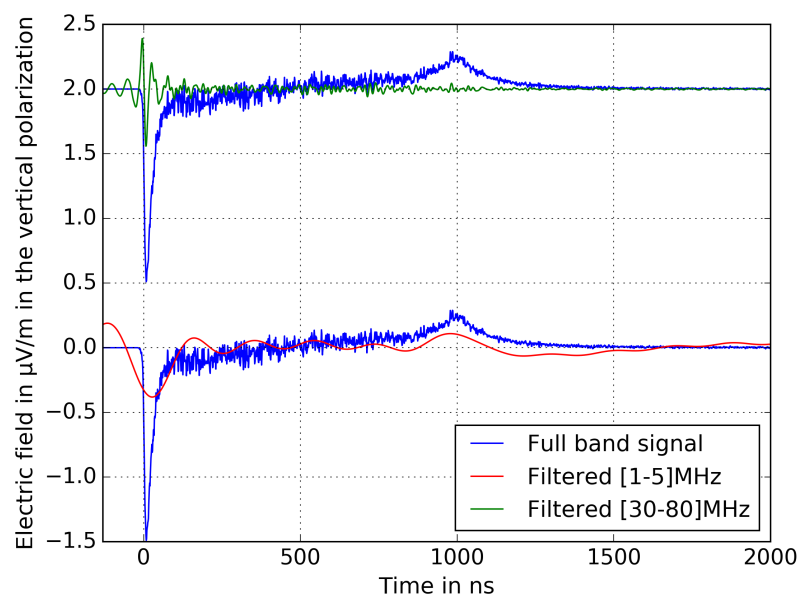

Figure 1: Electric field as a function of time obtained with a SELFAS simulation for a vertical shower at $0.1 \mathrm{EeV}$ : the associated filtered response in different bands showing that the two pulses can be seen in the range $[1-5] \mathrm{MHz}$.

we present the objectives of the EXTASIS project, our instrumental setup and our first results.

\section{Instrumental setup}

Currently, the EXTASIS project is based on low frequency antennas triggered by scintillators: two low frequency antennas directly triggered via cables, and five antennas for which the trigger is distributed over a network (see figure 2), and is supported by the CODALEMA experiment $[11,12]$.We know that the particle detector (scintillator) detects only cosmic ray events, because it is sensitive only to the particles arriving at ground. When at least 5 out of the 13 scintillators 
are fired in a given time window, a master trigger is built by a multiplicity card and sent to a dedicated datation station which dates by GPS the event, to the compact array and to the low frequency antennas. For the two cabled antennas, the timing is made by this dedicated GPS station, and data are acquired thanks to a digital oscilloscope (PicoScope). The devices are classical Butterfly antennas, a dual polarization (East-West and Vertical, since the SDP is expected to be also vertically polarized) active antenna using a bow-tie shaped rod as radiating element, with an adapted low noise amplifier to the band [2-6] MHz. They are disposed at equal distance from the scintillators center on a $9 \mathrm{~m}$ high mast. As suggested by NEC simulations, the losses in ground are minimized and the signal to noise ratio in the range [2-6] $\mathrm{MHz}$ is upper than $10 \mathrm{~dB}$ at $9 \mathrm{~m}$ height. The $2.10 \mathrm{~m}$ long dipoles composing the Butterfly antennas are considered as short when compared to the measured wavelengths. Each dipole feeds a low noise amplifier (LNA) whose input impedance is adapted to the dipole. The output signal of the LNA is transported by a cable to a radio frequency chain, followed by an analogical chain composed of filters. The signals of both polarizations feed the 2 inputs of a digital oscilloscope serving as a digitizer and driven by a dedicated acquisition software hosted by a distant PC. The oscilloscope sampling time bin is $2 \mathrm{~ns}$ and the traces contain 20,000 time bins, so the trace is recorded over a total time of $40 \mu \mathrm{s}$. Moreover, the two cabled low frequency antennas are supplemented by 5 network-connected low frequency antennas. These antennas are physically the same as the cabled antennas, and are also externally triggered by the scintillators. They are networkconnected due to technical limitations, and the trigger is thus distributed over an Ethernet network. When the trigger is pro-

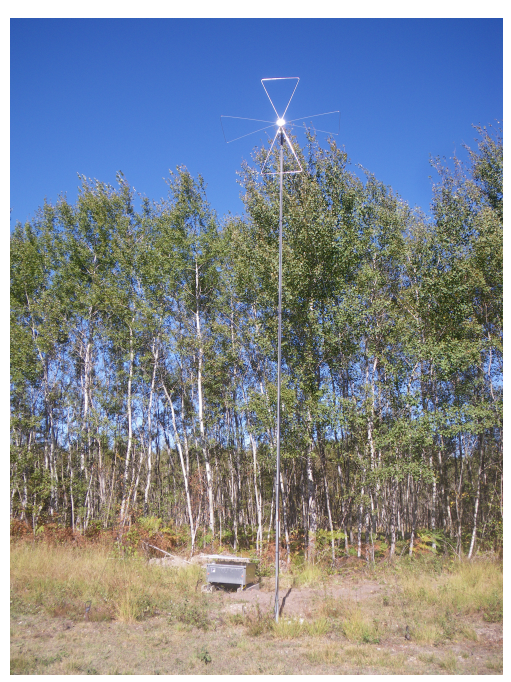

Figure 2: Photography of a low frequency antenna. duced by the particle detectors, it is sent to the GPS datation station which duplicates the trigger signal and sends it to an emission chassis which distributes the trigger signal over Ethernet connexion to the five concerned low frequency antennas composed of a reception chassis. First, the 5 network-connected low frequency antennas had an East-West (EW) and a vertical polarization, but to provide the detection of the low frequency counterpart, we have decided to replace the vertical polarization by an EW high frequency polarization: thus, the actual EXTASIS apparatus is composed of one Butterfly antenna in EW polarization at $9 \mathrm{~m}$ high (EXTASIS Low Frequency (LF)) working in the range $[1-6] \mathrm{MHz}$ and one Butterfly antenna in EW polarization at $1.5 \mathrm{~m}$ (EXTASIS High Frequency (HF)) working in the range $[20-200] \mathrm{MHz}$, and digitized by the same oscilloscope. The electronic modules of the reception chassis is located below the EXTASIS HF antenna. The two EXTASIS antennas are supplemented by one standalone antenna (SA, CODALEMA HF) working in the range $[20-200] \mathrm{MHz}$. The objective is to observe a pulse in the associated CODALEMA HF, observed the same pulse in the EXTASIS HF and use the timing of the pulse to find it in the EXTASIS LF, allowing us to affirm that the transients seen in LF are evidence of the low frequency counterpart of the radio signal in cosmic ray induced air showers. 


\section{First results}

\subsection{Low frequency sky}

Figure 3.(a) presents the atmospheric noise temperature in function of the frequency for the site of Nançay. In our case, the studied frequency range is dominated by the atmospheric noise due to the high brightness temperature of the atmosphere at these frequencies, which grows considerably during the night, but also by man-made noise. Figure 3.(b) shows a time-frequency diagram, which is a daytime power density spectrum for one low frequency antenna, and highlights the ambient noise variation during one day, and a day/night dependence can be seen, confirming that our low frequency antennas are sensitive to the day/night variation of atmospheric noise temperature as predicted and shown on figure 3.(a). The vertical black-dashed lines represent the sunrise and sunset, and delimit two regions: the "night region" where our instrument is not efficient, and the "day region", where our instrument is efficient, and where we expect to detect a low frequency signal due to a cosmic ray air shower. We can also see a clear man-made emitter at around 7.5 MHz. From

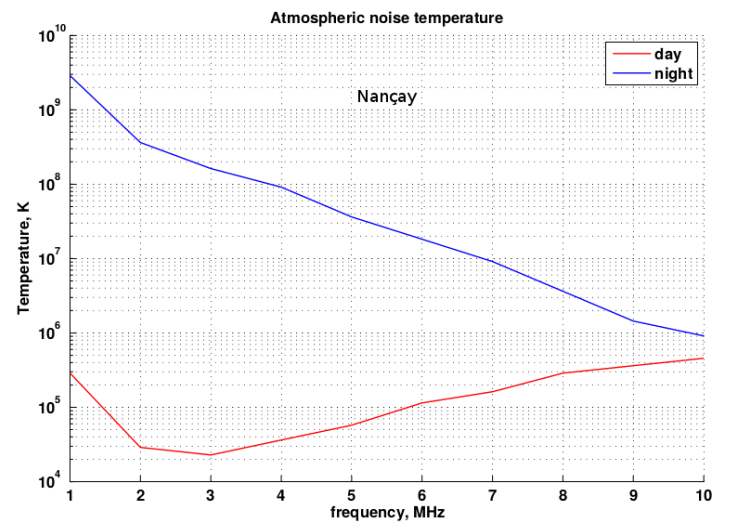

(a)

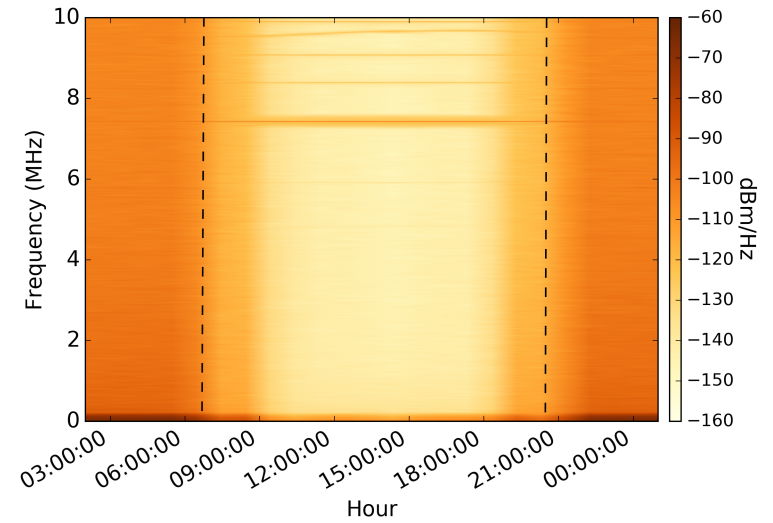

(b)

Figure 3: Left: atmospheric noise temperature in function of the frequency for the site of Nançay. Right: time-frequency diagram of a low frequency antenna. See text for more details.

this result, we have decided to focus our study on daytime, and particularly in the [1.7 - 3.7] $\mathrm{MHz}$ frequency domain, which is the cleanest band below $10 \mathrm{MHz}$.

\subsection{Low frequency signal detections}

First, the selection of low frequency events with a significant signal was done with an amplitude threshold. With this method, we have detected one low frequency event involving $2 \mathrm{LF}$ antennas. After a comparative study between three detection methods (amplitude threshold, wavelet analysis and LPC), we have chosen to use the LPC (linear predictive coding) method [13, 14]. With this method, we are more sensitive to events with a small signal to noise ratio, as shown in figure 4. This event is seen in coincidence by CODALEMA and EXTASIS, and the arrival direction reconstructions are in good agreement: $\theta_{L F}=31.1^{\circ}, \Phi_{L F}=146.1^{\circ}, \theta_{S A}=40.6^{\circ}, \Phi_{S A}=145.2^{\circ}$ and $\theta_{S C}=32.4^{\circ}, \Phi_{S C}=144.1^{\circ}$. One can see that the transients are hardly visible on the filtered traces (figure 4.(a) and (b)), but that they begin to appear after the LPC processing (figure 4.(c) and (d)). 


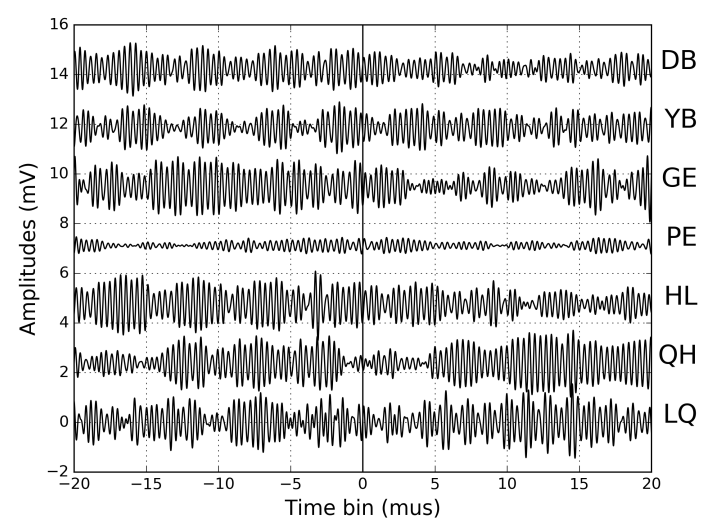

(a) Filtered [1.7 - 3.7] MHz LF signals.

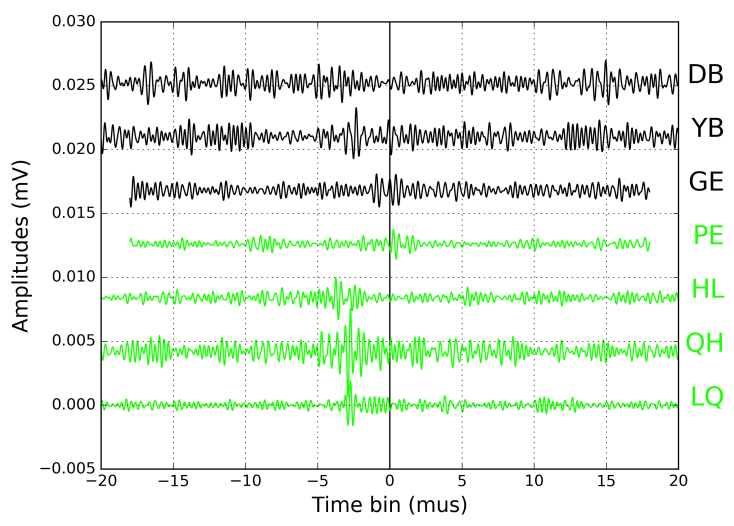

(c) Error of prediction on LF signals.

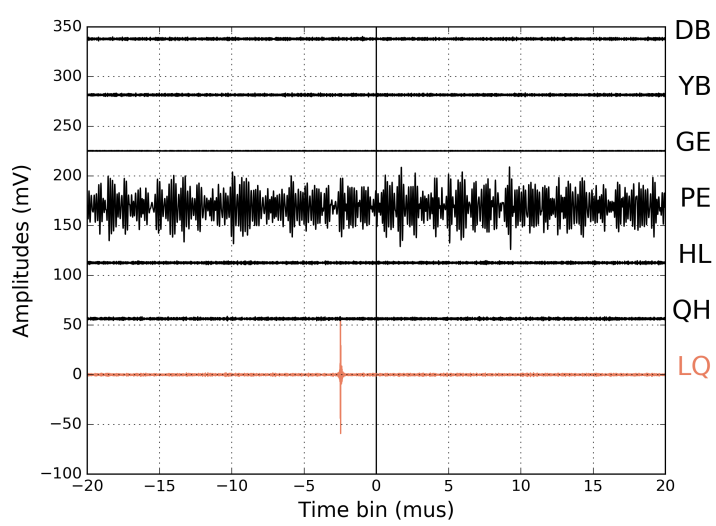

(b) Filtered $[30-80] \mathrm{MHz}$ HF signals.

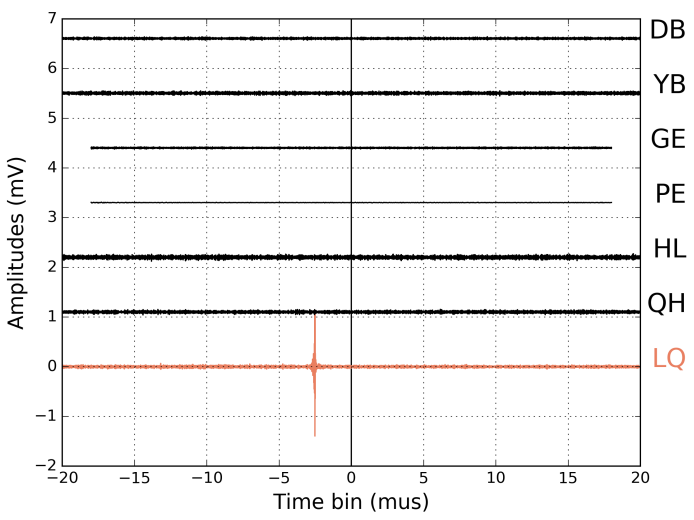

(d) Error of prediction on HF signals.

Figure 4: LF event seen with the EXTASIS instrument. From the top, left: Filtered LF signals, filtered HF signals, error of prediction on LF signals and on HF signals.

With SELFAS, we simulated this event, and made the radio core reconstruction, see [15] for more explanation on the method. The best core position is in $x=259 \mathrm{~m}$ and $y=-809 \mathrm{~m}$, and represented by a magenta square in figure 5. Figure 5 shows the map of a part of facilities in Nançay. Grey dots represent the standalone antennas, and the stars the low frequency antennas. The involved standalone antennas in the coincidence are represented by coloured circles indicating the order in which the signal is seen by the antennas, and the involved low frequency antennas are represented by green crosses. The low frequency antenna named LQ (see figures 4.(c) and (d)) presents a signal in both polarizations at the same position in the trace, particularly in the HF polarization, while the 3 other ones have only a signal in the LF polarization. A possible explanation is that the LQ antenna is close to the estimated shower core, as one can see on figure 5, and that the 3 other ones are very far away from it, hinting that the detection range at low frequency is higher than at high frequency. Making a precise simulation of the event thanks to the information from the radio reconstruction [15], it is shown in figure 6 which presents the simulated lateral distribution of the electric field 


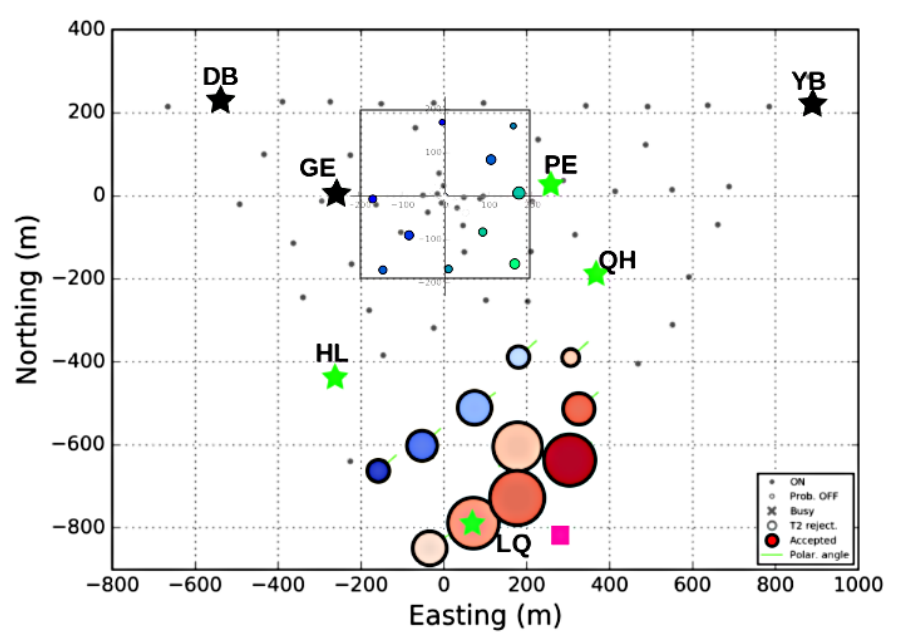

Figure 5: Map of a part of facilities in Nançay. Grey dots represent the standalone antennas, the square area with 13 circles represents the scintillators and the stars the low frequency antennas. The involved standalone antennas are represented by coloured circles indicating the order in which the signal is seen by the antennas, and the involved low frequency antennas are represented by green crosses. The estimated shower core is represented by a red square.

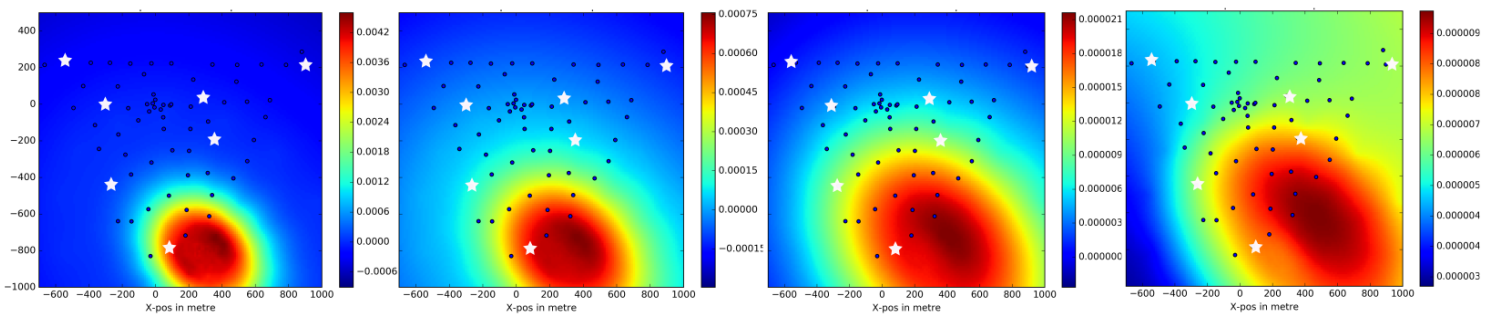

Figure 6: Lateral distribution of the electric field depending on the frequency range. From left to right: $[30-80] \mathrm{MHz},[10-30] \mathrm{MHz},[5-10] \mathrm{MHz}$ and [1 -5] MHz. White stars represent the low frequency antennas. At low frequency, the LF antennas very far away from the shower core begin to be in the detection zone and thus sensitive.

depending on the frequency band that at low frequency (figure 6 right), the LF antennas very far away from the shower core begin to be in the detection zone, explaining why the PE antenna situated at $850 \mathrm{~m}$ presents a signal in the low frequency band, and not in the high frequency band. Moreover, figure 7 presents the simulated power spectrum density as a function of frequency for the four involved LF antennas. We see that the PSD quickly drop in the classical band with the shower axis distances, while they decrease much more slowly in the LF band. This can explain also why LQ presents a signal in both polarizations while the three others present a signal only in the LF polarization. This result indicates that the detection range is larger at low frequency than at high frequency.

This finding is confirmed by another event figure 8, seen by four LF antennas. Three of them have a signal in both polarizations, while the farthest one from the shower core presents a signal only in the LF polarization. This result endorses our argument that detection range is better 


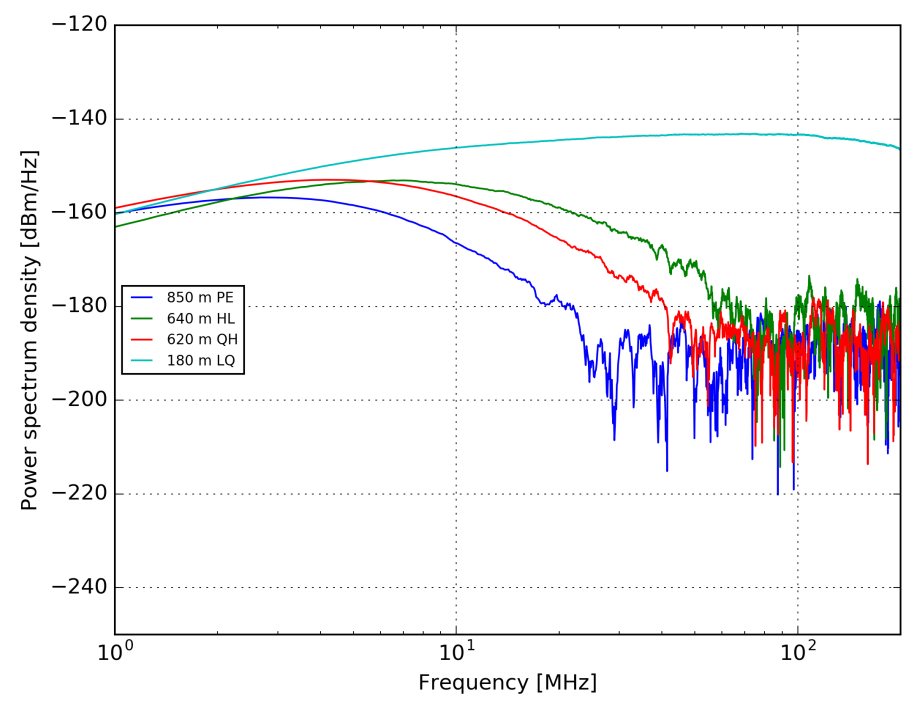

Figure 7: Simulated power spectrum density as a function of frequency for the four involved LF antennas.

at low frequency than at high frequency. The arrival direction reconstructions are again in good
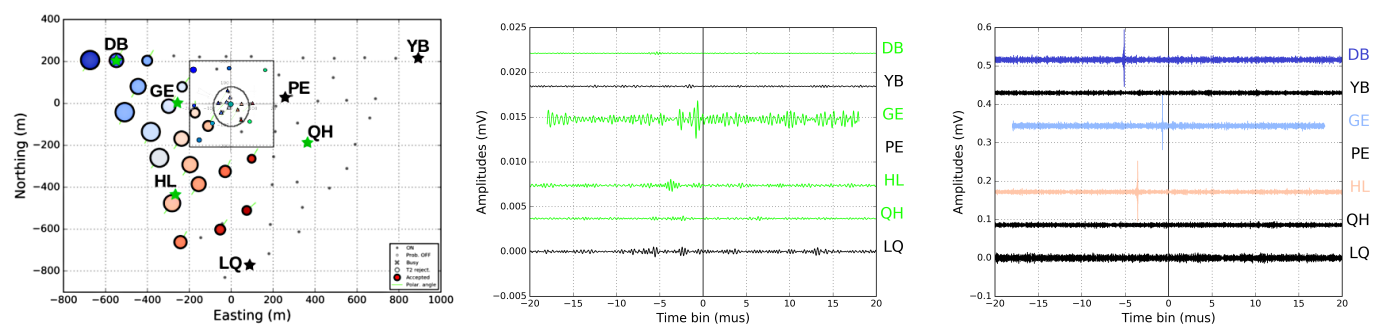

Figure 8: LF event seen with the EXTASIS instrument. From left: map of event (green crosses represent the involved low frequency antennas), the square area with 13 circles represents the scintillators and the triangles in the circular area represent the compact array, error of prediction on LF signals and error of prediction on HF signals.

agreement: $\theta_{L F}=66.8^{\circ}, \Phi_{L F}=155.0^{\circ}, \theta_{S A}=60.1^{\circ}, \Phi_{S A}=153.8^{\circ}$ and $\theta_{S C}=61.6^{\circ}, \Phi_{S C}=154.6^{\circ}$.

\section{Conclusion and outlooks}

Since the installation of the instrumental setup of EXTASIS experiment, the low frequency sky in Nançay has been explored, allowing us to understand the low frequency antenna environment, to put in evidence its behaviour and therefore to convince us that our low frequency instrument is functional.

In this proceeding, we have shown two low frequency events in coincidence with the particle detectors and the other radio instruments installed in Nançay, what is a clear evidence of a low frequency counterpart of the radio signal emitted by cosmic ray induced air showers. Moreover, these results 
seem to confirm the expectations, particularly that the detection range at low frequency is better than at high frequency.

Now that the timing of signal is verified thanks to the high frequency polarizations installed on the low frequency antennas, we need to switch to the previous instrumental setup version, and replace the high frequency polarization by a vertical polarization, in order to be sensitive to the sudden death signal which is expected also in this polarization.

\section{Acknowledgements}

We thank the Région Pays de la Loire for its financial support of the Astroparticle group of Subatech and in particular for its contribution to the EXTASIS experiment.

\section{References}

[1] F. D. Kahn et al. Radiation from Cosmic Ray Air Showers. Proceedings of the Royal Society of London A: Mathematical, Physical and Engineering Sciences, 289(1417):206-213, 1966.

[2] GA Askaryan. . J. Exp. Theor. Phys. 21 (1962) 658.

[3] A. Bellétoile et al. Evidence for the charge-excess contribution in air shower radio emission observed by the CODALEMA experiment. Astroparticle Physics, 69:50-60, September 2015.

[4] J. R. Prescott et al. Properties of radio emission of extensive air showers. In International Cosmic Ray Conference, volume 3 of International Cosmic Ray Conference, page 717, 1970.

[5] H. R. Allan et al. Frequency Spectrum of Air Shower Radio Pulses. Nature, 225:253-254, January 1970.

[6] T. J. Stubbs. Evidence of Radio Pulses at 2 MHz from Cosmic Ray Air Showers. Nature, 230:172-173, April 1971.

[7] J. H. Hough et al. Observation of Radio Pulses from Extensive Air Showers at 3.6 MHz. Nature Phys Sci, 232:14-15, July 1971.

[8] Vincent Marin et al. Simulation of radio emission from cosmic ray air shower with \{SELFAS2\}. Astroparticle Physics, 35(11):733 - 741, 2012.

[9] B. Revenu et al. Radio emission from the air shower sudden death. ArXiv e-prints [1211.3305] and ICRC13, November 2012.

[10] D. Garcia-Fernandez et. al. See proceedings for this conference.

[11] R.Dallier et al. Recent results from CODALEMA and the Nançay radio facilities related to cosmic-ray measurements. EPJ Web Conf., 135:01002, 2017.

[12] B.Revenu et. al. See proceedings for this conference.

[13] D. Ardouin et al. Radioelectric field features of extensive air showers observed with CODALEMA. Astroparticle Physics, 26(4):341 - 350, 2006.

[14] S. Valcares. Cosmic ray properties from the electric fields measured by the CODALEMA experiment. Theses, Université de Nantes, September 2008.

[15] L. Martin et. al. See proceedings for this conference. 